\title{
Unilateral pulmonary hemorrhage caused by negative pressure pulmonary edema.
}

\author{
Hyung Park ${ }^{1}$, Seong Park $^{1}$, Un Woo ${ }^{1}$, Sang Cho ${ }^{1}$, Woo Jeon ${ }^{1}$, and Woo Shin ${ }^{1}$ \\ ${ }^{1}$ Hanyang University Medical Center
}

September 21, 2020

\begin{abstract}
We experienced a unilateral alveolar hemorrhage associated with negative pressure pulmonary edema that occurred after long history of asthma patient bit the endotracheal tube during posture change at the end of surgery. We describe herein perioperative and intensive care management of unilateral pulmonary hemorrhage caused by biting endotracheal tube.
\end{abstract}

\section{INTRODUCTION}

Diffuse alveolar hemorrhage (DAH) is characterized by damage to the alveolar microvessels and accumulation of red blood cells in the alveoli. DAH is a syndrome in which respiratory failure occurs due to hemoptysis, anemia, and hypoxemia. ${ }^{1,2}$ Pulmonary imaging may be important in the diagnosis. The most frequent causes of DAH are forms of systemic vasculitis such as anti-glomerular basement antibodies (GBM Ab), granulomatosis with polyangitis, microscopic polyangitis, capillaritis, vasculitis, or complications that can occur in patients with diseases such as Wegener's granulomatosis, Goodpasture's syndrome, and systemic lupus erythemasus. ${ }^{3}$

DAH may also be associated with surgery and anesthesia. It has been reported that, especially in young, healthy, and muscular men with upper respiratory tract obstruction during anesthesia, negative pressure in the lungs is created, resulting in negative pressure pulmonary edema (NPPE) and, in rare cases, bilateral pulmonary hemorrhage. ${ }^{3}$ It has also been reported that one-sided alveolar or pulmonary hemorrhage may occur in cases of the development of negative intrathoracic pressure owing to upper respiratory tract obstruction or administration of a large amount of fluid to a patient in the lateral position, or in the presence of a disease such as right atrial failure. ${ }^{3,4} \mathrm{DAH}$, although often spontaneously resolving, can sometimes lead to fatal complications.

In the case we report here, we attempted to remove the patient's endotracheal tube under deep anesthesia at the end of surgery in order not to irritate the airway in a patient who had lived with asthma for many years. However, we report this case with a literature review and informed consent to publication was obtained from the guardian because we experienced a unilateral DAH associated with NPPE that occurred after a short period of airway obstruction after the patient bit the intubated endotracheal tube during posture change.

\section{CASE REPORT}

A 78-year-old male patient diagnosed with spinal stenosis was admitted to undergo unilateral laminectomy with bilateral decompression. The patient had been diagnosed with hypertension four years earlier and asthma more than 70 years earlier, and was prescribed fluticasone furoate and vilanterol inhalers for the treatment of asthma; however, these medications were not used consistently. The results of pulmonary function tests were FVC $2.53 \mathrm{~L}(61 \%), \mathrm{FEV}_{1} 1.37 \mathrm{~L}(52 \%)$, and $\mathrm{FEV}_{1} / \mathrm{FVC} 54$, and chest radiographs showed subsegmental atelectasis in the left lower lobe and mild cardiomegaly (Fig. 1). Transthoracic 
echocardiography performed prior to surgery showed concentric left ventricular hypertrophy, trivial mitral regurgitation, and a mild relaxation abnormality, with other findings within normal limits. As premedication, hydrocortisone $100 \mathrm{mg}$ was administered on the morning of surgery. Lung sounds confirmed after the patient entered the operating room were normal, and anesthesia was initiated after prophylactic administration of salbutamol inhalants. General anesthesia was induced with thiopental $250 \mathrm{mg}$ and fentanyl $100 \mathrm{mcg}$, and the patient intubated without special difficulty after administration of rocuronium $50 \mathrm{mg}$. Anesthesia was maintained at $50 \% \mathrm{~N}_{2} \mathrm{O}$ and $5-7 \%$ desflurane. The operation was performed with the patient in the prone position, and there were no special events during the operation. The arterial blood gas analysis (ABGA) performed during the operation showed $\mathrm{FiO}_{2} 0.5$ to $\mathrm{pH} 7.420, \mathrm{pO}_{2} 242.5, \mathrm{pCO}_{2} 36.7$, and base excess - 0.6, and hemoglobin level was $12.2 \mathrm{~g} / \mathrm{dl}$. At the end of the operation, sugammadex $200 \mathrm{mg}$ was administered for reversal of muscle relaxation, the patient's position was changed from prone to supine under deep anesthesia, and the bispectral index (BIS) score at that time was in the 40. Subsequently, several aspiration attempts were made to remove the secretions from the trachea and oral cavity in order to manage the tracheal tube, but the patient was clenching his teeth and did not open his mouth. At this time, airway obstruction occurred for several minutes, making ventilation difficult. Oxygen saturation decreased by $40 \%$ on pulse oximetry even though $50 \%$ of oxygen were provided and the concentration of inhalation anesthetics were not reduced. An additional $200 \mathrm{mg}$ sugammadex was administered owing to severe airway obstruction caused by the patient's clenching his teeth. After a few minutes, the patient stopped clenching his teeth, but a large amount of bloody discharge from the endotracheal tube was observed. Furosemide $40 \mathrm{mg}$ was administered immediately based on suspected negative pressure pulmonary edema due to airway obstruction. The patient was ventilated with $100 \%$ oxygen, but the oxygen saturation was maintained at $90 \%$ or less. Chest imaging (Fig. 2) performed after transfer to the intensive care unit revealed haziness of the entire right lung, and bronchoscopy (Fig. 3), showed bloody secretions with red bubbles in the right lung. However, no active bleeding or endobronchial lesions were found. ABGA performed after transfer to the intensive care unit showed $\mathrm{pH} 7.30, \mathrm{pO}_{2} 72.9, \mathrm{pCO}_{2} 40.6$, and $\mathrm{HCO}_{3} 20.1$, $\left(\mathrm{FiO}_{2}\right.$ 1.0) with hemoglobin of $11.4 \mathrm{~g} / \mathrm{dl}$. On the day of surgery, norepinephrine and vasopressin were used because the patient's blood pressure decreased in the intensive care unit. Tranexamic acid and methylprednisolone were administered for pulmonary hemorrhage and pulmonary edema. Extubation was performed on the $8^{\text {th }}$ postoperative day (POD) and the patient was transferred to the general ward on the $10^{\text {th }}$ POD. On the subsequent chest radiography, most of the haziness of the right lung had disappeared (Fig. 4). The patient was discharged without any complications after a week after moving to the general ward.

\section{DISCUSSION}

The patient in this case had been diagnosed with asthma many years previously. To remove the endotracheal tube gently without irritating the airway following the surgery, we changed the patient's posture under deep anesthesia. During this process, the patient severely bit the tube and we were unable to provide ventilation for several minutes. Negative pressure pulmonary edema occurred owing to severe negative intrathoracic pressure due to airway obstruction caused by the severe biting of the endotracheal tube, and a large quantity of bloody secretion was released into the intubation tube. At first, NPPE was suspected, and treatment was actively performed, including fluid restriction, diuretic administration, exhalation positive breathing, and $100 \%$ oxygen administration. Chest imaging performed after transfer to the intensive care unit showed diffuse haziness in the right lung field and persistent hemoptysis. Bronchoscopy findings showed no abnormal airway damage with the exception of bloody secretion in the bronchus or airways.

According to Mondoni et al. ${ }^{5}$ early bronchoscopy helps detect bleeding sources. They suggested that if there is rebleeding, the bronchoscopy must be performed again to examine the damaged airway area. Hemoptysis caused by damage to the airways due to temporary negative pressure is reported to naturally cease over time.

Shankar et al. ${ }^{6}$ reported airway bleeding and hemoptysis caused by temporary intrathoracic negative pressure resulting in airway damage from airway obstruction due to various causes. This may be misdiagnosed as pulmonary edema. The authors suggested that hemoptysis may be caused by aspiration pneumonitis, 
airway injury, polyps, arteriovenous malformation, and tumors.

Prost et al. ${ }^{7}$ reported that NPPE was a rare $(5 \%, 6 / 112)$ cause of DAH. Contou et al. ${ }^{1}$ surveyed 149 patients in whom DAH was suggested over 36 years (1980-2015) and found that 18 cases (12\%) of DAH occurred in 15 patients through NPPE. According to the study, almost 17 cases (94\%) showed acute respiratory failure with no other organ failure or circulatory failure. Hemoptysis was found in 17 out of 18 cases (94\%) and was the most common symptom, but in only one case was more than $200 \mathrm{ml}$ per day observed. Anemia was observed in 10 cases $(56 \%)$ and the average hemoglobin reduction was $1.5 \mathrm{~g} / \mathrm{dl}$. It was reported that 9 cases (50\%) satisfied the DAH triad (hemoptysis, anemia, and pulmonary infiltrates). In this case, hemoptysis was the main symptom and anemia was not severe. The authors reported that 16 cases (89\%) showed alveolar or interstitial opacity of both lungs, and only two cases (11\%) demonstrated alveolar or interstitial opacity in the right lung. In the present case, diffuse opacity was observed in the right lung.

Schwarz et al. ${ }^{8}$ reported pulmonary hemorrhage after NPPE in surgical patients for the first time, and Goldenberg et al. ${ }^{9}$ reported two patients with tricuspid and pulmonary insufficiency among six post-laryngospasm NPPE patients. Dolinski et al. ${ }^{3}$ reported pulmonary hemorrhage in patients with mild tricuspid regurgitation due to increased pulmonary artery pressure after NPPE. In this case, the patient had evidence of trivial MR in the transthoracic echocardiography performed before surgery, but the clinical symptom was not specific. It is thought that a more detailed examination is needed to determine whether this mild MR was the cause of unilateral DAH in this case, but such an examination was not performed in this patient. In 2016, Han et al. ${ }^{10}$ reported negative pressure pulmonary hemorrhage that occurred after laryngospasm in the postoperative period. In 2016, Choe et al. ${ }^{11}$ reported DAH after trans-femoral cerebral angiography in patients taking clopidogrel, and there is a potential for DAH associated with neuro-intervention in patients with hemoptysis and respiratory failure. It was argued that patients who are taking anticoagulants should carefully taper off the medicine prior to surgery and then begin it again during or after surgery. In addition, the author stated that it is better to protect less damaged lungs through active lung management such as oxygen therapy, bronchodilators, endotracheal intubation, and mechanical ventilation management in the treatment of patients with DAH.

NPPE by acute upper respiratory tract obstruction has been reported in about $11 \%$ of cases. ${ }^{12}$ Risk factors for postoperative obstruction include obesity, short neck, obstructive sleep apnea, upper respiratory surgery, hypertrophy, and difficulty in tracheal intubation. While any factor that can cause gastrointestinal obstruction can cause NPPE, the most common cause of NPPE during anesthesia is postoperative laryngospasm.

It is reported that NPPE occurs within a few minutes of airway obstruction and improves quickly after a day or two with active treatment. Steroids, diuretics, and bronchodilators are known to help, but their actions in these cases are not clear. ${ }^{12}$

Papaianou et al. ${ }^{13}$ reported that hemoptysis caused by NPPE, which occurs during obstruction of the upper airways, such as cases of laryngospasm, may be caused by negative-pressure tracheobronchial injury or alveolar-capillary disruption. It is also said that tension applied to the pulmonary-capillary wall may be caused by alveolar-capillary membrane rupture. The authors stated said that administration of succinylcholine (Sch) $80 \mathrm{mg}$ and administration of an unconsciousness inducing agent can prevent airway obstruction caused by laryngospasm. ${ }^{13}$ Both laryngospasm and biting were possible causes of the patient's airway obstruction in the present case. The authors chose sugammadex for muscle relaxation reversal instead of Sch in this case, focusing more on the visible biting rather than laryngospasm as the cause of airway obstruction, and the patient quickly stopped biting and was able to undergo ventilation.

Young male athletes are known to be at risk, and the syndrome occurs rarely in patients with endotracheal intubation, but may also occur if the patient bites the endotracheal tube during waking. ${ }^{14}$ Additional causes include excessive endotracheal or laryngeal aspiration, as well as abnormal positioning of the mask. However, there are few reports of pulmonary hemorrhage accompanied by NPPE due to upper respiratory tract obstruction. ${ }^{1,8}$

Schwartz et al. ${ }^{8}$ reported that at the end of surgery, negative intrathoracic pressure caused by upper airway 
obstruction after extubation causes hypoxic respiratory failure. The authors noted that post-anesthetic laryngospasm, strangulation, hanging, foreign body airway obstruction, and OSA may be factors associated with NPPE.

Contou et al. ${ }^{1}$ reported that the mechanisms of development of NPPE were negative pressure formation in the thoracic cavity, increased hydrostatic pressure in systemic and pulmonary capillaries, and mechanical stress in the alveolar capillaries.

According to Contou et al., ${ }^{1}$ first, $-2^{\sim}-5 \mathrm{cmH}_{2} \mathrm{O}$ intrathoracic negative pressure $\left(-100 \mathrm{cmH}_{2} \mathrm{O}\right.$ in severe cases) increases venous return from peripheral blood vessels to the right atrium, simultaneously increasing pulmonary blood flow, increasing trans-mitral capillary pressure, and increasing pulmonary capillary and alveolar cavity pressure and stress. The hydrostatic pressure rises. These changes increase the trans-capillary pressure gradient, and the fluid exudes into the interstitial space. The author also reported that elevated central venous pressure interferes with lymphatic blood circulation.

Second, according to Contou et al., ${ }^{1}$ the systemic pressure increases owing to the secretion of norepinephrine due to hypoxemia, hyper carbon dioxide, and anxiety, which increases the amount of pulmonary blood flow. These effects reduce right ventricular dilatation, ventricular wall movement, and cardiac output. As the vascular resistance of the whole body increases, the resistance of the left ventricular wall increases, and the left ventricle prevents the blood flow.

Lastly, the authors reported that effusion from capillaries due to physical alveolar-capillary membrane damage caused alveolar edema and bleeding.

Unilateral pulmonary edema is a very rare complication. This complication is mainly reported in cases of heart disease such as severe mitral insufficiency or congestive heart failure, and there are few reports in patients without heart disease. The syndrome can be caused by prolonged lying on the lateral side or by excessive administration of fluids in this position, and can occur after treatment in cases such as pleural effusion or pneumothorax, or after re-expansion. MacLeod syndrome, unilateral pulmonary agenesis, talc pleurodesis, trauma, epilepsy, upper airway obstruction, pulmonary venous obstruction from mediastinal fibrosis, post-lobectomy, unilateral mainstem intubation, nitrogen mustard, and heroin-related pulmonary edema, and pregnancy may all be causes of the syndrome. ${ }^{15}$ The patient in the present case was not in the lateral position and had no specific trigger except for asthma from which he had been suffering for a long time.

As such, negative unilateral pulmonary edema and unilateral pulmonary hemorrhage may result from many causes. In patients with acute respiratory failure with hemoptysis and unilateral alveolar opacity, if clinical findings are not consistent with pneumonia, it is important to determine the presence of heart disease. Rapid initiation of active treatment is important.

In summary, DAH is mainly caused by airway obstruction after surgery in young and healthy male athletes. The patient in the present case demonstrated acute respiratory failure accompanied by hemoptysis and alveolar opacity on both sides, along with bilateral bleeding from both sides and no bronchial damage. It is known that in older individuals, there is a high possibility that there will not be enough negative pressure to destroy the pulmonary capillaries, but the patient in this case was an elderly male patient who had been diagnosed with asthma 70 years earlier, considered to be at increased risk and with damaged sensitivity to the endothelial tissue of the bronchioles.

In a 78-year-old man treated with asthma for a long time, change to the supine position at the end of general anesthesia resulted in severe hypoxic respiratory failure, increased airway pressure, and intra-tracheal tube after acute upper respiratory obstruction owing to severe endotracheal tube biting. The patient experienced a large amount of bloody secretions. Ultimately, the patient was discharged without complications owing to appropriate treatment for negative pressure pulmonary hemorrhage that occurred after endotracheal tube biting. Anesthesiologists should take care not to induce airway irritation during emergence of asthma, because DAH accompanied by NPPE was caused in this case by airway irritation in an older patient with 
asthma without known risk factors.

\section{ETHICAL APPROVAL}

Not applicable

\section{CONSENT FOR PUBLICATION}

Written informed consent was obtained from the guardian for publication of this case reports. Families of patients for cooperating to the investigations.

FUNDING INFORMATION No funding supported to this work

\section{CONFLICT OF INTEREST}

No potential conflict of interest relevant to this article was reported

\section{AUTHOR CONTRIBUTIONS}

HJ Park made administrative, technical, or material support and did the literature searches. UT Woo and SH Park had full access to all of the laboratory data of a patient and the accuracy of the data analysis. WJ Jeon involved in case report manuscript design, analysis, and interpretation of picture and laboratory data; drafted the manuscript. SY Cho and WJ Shin involved in critical revision of the manuscript for important intellectual content. All of the authors have reviewed the manuscript and accepted it in the current form for publication.

\section{ORCID}

Hyung Joon Park - 0000-0002-3114-5355,

Seong Ho Park - 0000-0003-3664-2251

Un Tak Woo - 0000-0002-3492-2199,

Sang Yun Cho - 0000-0002-6593-1960

Woo Jae Jeon - 0000-0002-9662-4197,

Woo Jong Shin - 0000-0002-3786-2267

\section{ACKNOWLEDGMENTS}

None

\section{REFERENCES}

1. Contou D, Voiriot G, Djibre M, Labbe V, Fartoukh M, Parrot A. Clinical Features of Patients with Diffuse Alveolar Hemorrhage due to Negative-Pressure Pulmonary Edema. Lung . 2017;195(4):477-487.

2. Hao D, Basnet S, Melnick S, Kim J. Negative pressure pulmonary edema-related diffuse alveolar hemorrhage associated with Sevoflurane and cigarette smoking. J Community Hosp Intern Med Perspect . 2019;9(3):247-251.

3. Dolinski Sylvia Y, M.D., MacGregor Drew A, M.D., Scuderi Phillip E, M.D. Pulmonary Hemorrhage Associated with Negative-pressure Pulmonary Edema. Anesthesiology: The Journal of the American Society of Anesthesiologists . 2000;93(3):888-890.

4. Attias D, Mansencal N, Auvert B, et al. Prevalence, characteristics, and outcomes of patients presenting with cardiogenic unilateral pulmonary edema. Circulation . 2010;122(11):1109-1115.

5. Mondoni M, Carlucci P, Cipolla G, et al. Bronchoscopy to assess patients with hemoptysis: which is the optimal timing? BMC Pulm Med . 2019;19(1):36. 
6. Bhavani-Shankar K, Hart NS, Mushlin PS. Negative pressure induced airway and pulmonary injury. Can J Anaesth . 1997;44(1):78-81.

7. de Prost N, Parrot A, Cuquemelle E, et al. Diffuse alveolar hemorrhage in immunocompetent patients: etiologies and prognosis revisited. Respir Med . 2012;106(7):1021-1032.

8. Schwartz DR, Maroo A, Malhotra A, Kesselman H. Negative pressure pulmonary hemorrhage. Chest . 1999;115(4):1194-1197.

9. Goldenberg JD, Portugal LG, Wenig BL, Weingarten RT. Negative-pressure pulmonary edema in the otolaryngology patient. Otolaryngol Head Neck Surg . 1997;117(1):62-66.

10. Han IS, Han BM, Jung SY, Yoon JR, Chung EY. Negative Pressure Pulmonary Hemorrhage after Laryngospasm during the Postoperative Period.Acute Crit Care . 2018;33(3):191-195.

11. Choe YS, Kim NY, Lee AR. Diffuse Alveolar Hemorrhage in Patients Undergoing Neurointervention: A Case Report. Anesthesiology and pain medicine . 2016;6(5):e33979-e33979.

12. Casoni GL, Tomassetti S, Coffa A, Ravaglia C, Poletti V. Negative pressure pulmonary hemorrhage induced by a candy. Am J Emerg Med . 2010;28(1):112 e113-115.

13. Papaioannou V, Terzi I, Dragoumanis C, Pneumatikos I. Negative-pressure acute tracheobronchial hemorrhage and pulmonary edema. J Anesth . 2009;23(3):417-420.

14. Liu EH, Yih PS. Negative pressure pulmonary oedema caused by biting and endotracheal tube occlusiona case for oropharyngeal airways. Singapore Med $J$. 1999;40(3):174-175.

15. Agarwal R, Aggarwal AN, Gupta D. Other causes of unilateral pulmonary edema. Am J Emerg Med . 2007;25(1):129-131.

Figure Legends

Figure 1. Preoperative chest radiograph

Figure 2. Postoperative chest radiograph at the intensive care unit

Figure 3. Postoperative bronchoscopic examination

Figure 4. Postoperative chest radiographic examination at $8^{\text {th }}$ postoperative day 

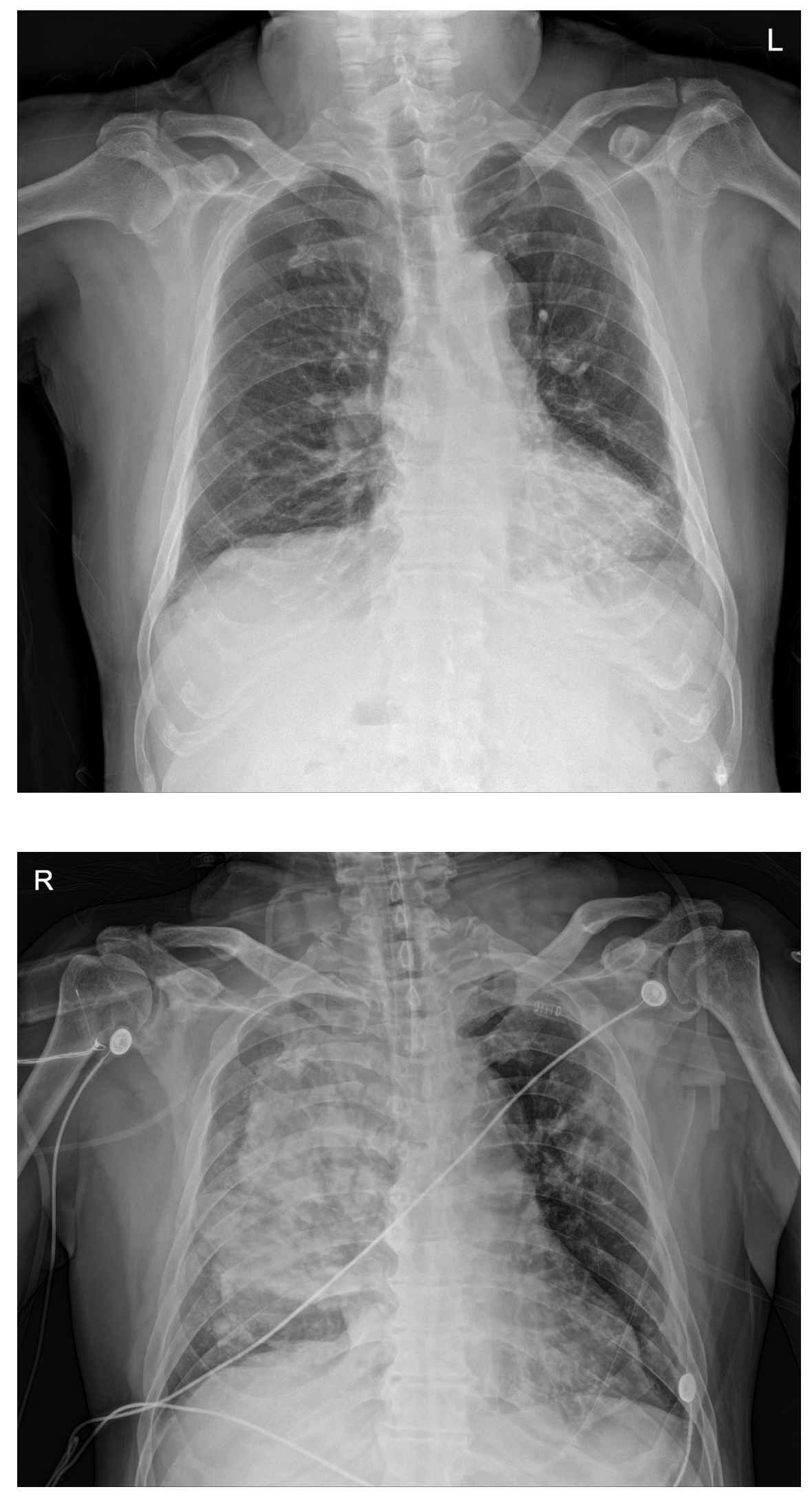

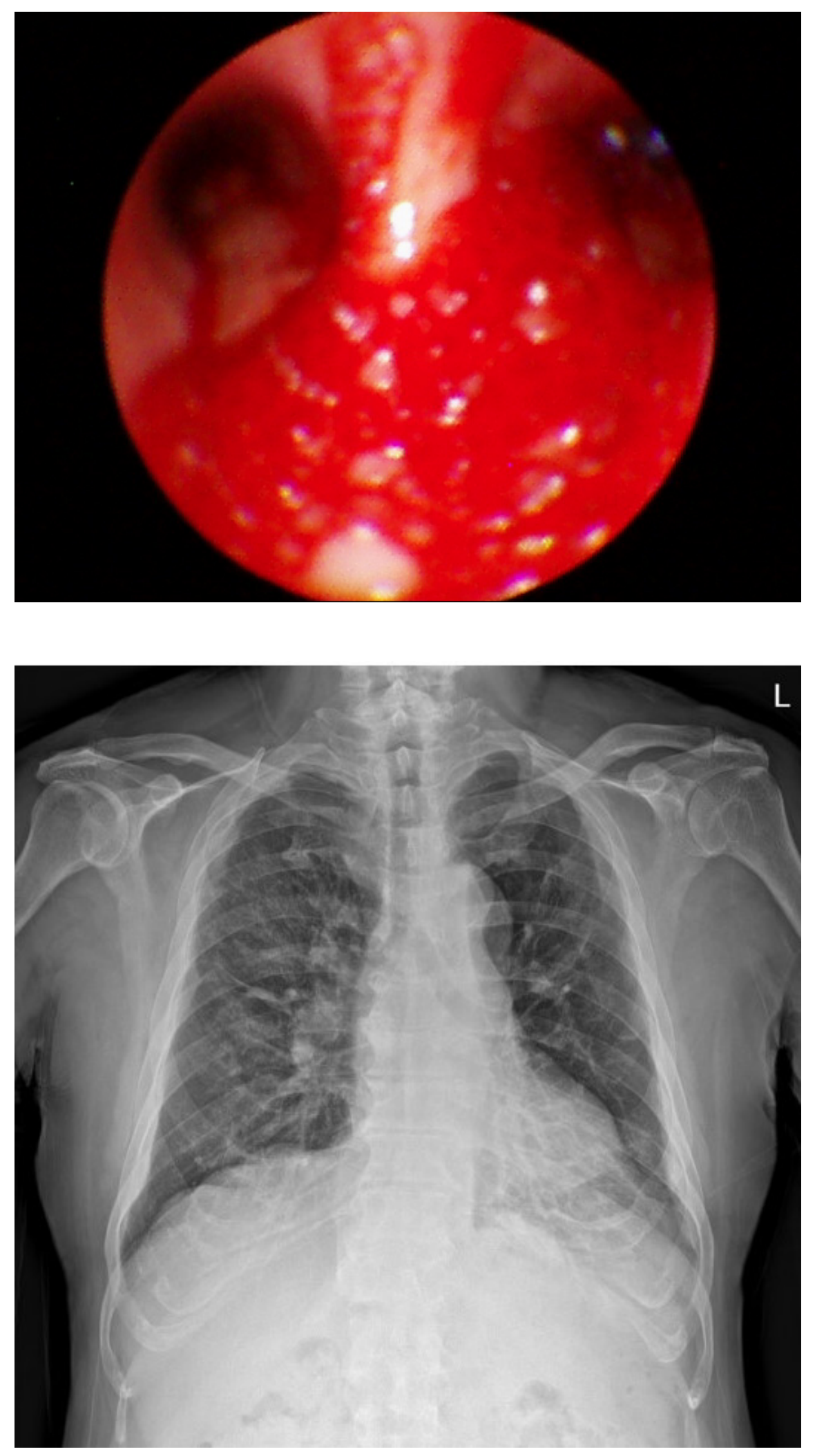